\title{
Piloting a holistic information culture program: The experience of CETYS Universidad system of libraries
}

\author{
Juan D. Machin-Mastromatteo, Omar Beltrán and Jesús Lau
}

\begin{abstract}
This article was originally published as: Machin-Mastromatteo, J. D., Beltrán, O., y Lau, J. (2014). Piloting a holistic information culture program: The experience of CETYS Universidad System of Libraries. In S. Kurbanoğlu, S. Špiranec, E. Grassian, D. Mizrachi y R. Catts (Eds.). Information Literacy. Lifelong Learning and Digital Citizenship in the 21st Century; Communications in Computer and Information Science, 492, 350-360. Cham: Springer.
\end{abstract}

The final publication is available at: $\underline{\text { http://link.springer.com/chapter/10.1007\%2F978-3-319-14136-7 } 37}$

\begin{abstract}
This article presents the staff, structure, methods and preliminary results from the pilot of a holistic information literacy program developed in the System of Libraries of CETYS Universidad in Mexico. 'Information Culture Development' (ICD) is driven by action research (AR) and the concept of information culture (IC), comprised of information literacy (IL), digital literacy (DL), and research competences. ICD aims at developing these competences and supporting reflection and improvement upon university practices related to curriculum, teaching, and research. ICD's initiatives and products were divided into four axes: a) curriculum and learning support, b) information and digital literacies development, c) research and scientific communication support, and d) evaluation and communication of results. ICD's pilot involved workshops and activities framed within an AR perspective and a mixed methods approach. Preliminary results determine the success of activities with academics and students regarding their strengths and weaknesses in IC-related competencies.
\end{abstract}

Keywords: Information literacy, digital literacy, information culture, action research, higher education, CETYS Universidad.

\section{Introduction}

Information literacy (IL) indicates the capacity to develop competencies for locating, retrieving, evaluating, and using information. Several academics and experts have written about IL, generally associating it with problem solving, decision making, emancipation and the exercise of citizenship, the overcoming of different forms of oppression and divides, critical thinking, and lifelong learning [1]. Many of these purposes fulfilled by an IL initiative are part of the worldwide tendencies seeking to support learning processes and are present in the vision of CETYS Universidad [2]. Hence, the IL program described in this document is grounded in both national [3] and international guidelines [4], as well as in sound research agendas [1], [5]. Apart from IL, it is also a priority for the program to develop digital literacy (DL), which refers to the 'proper use' of Information and Communication Technologies (ICTs) for teaching and learning; and institutional needs also point toward addressing academic communication and research competences [6]. The latter area has been currently 
explored and enhanced at the institution. Therefore, this initiative was conceived as a transversal axis and a driving force for the support of reflection and improvement upon practices related to curriculum, teaching and research activities within the institution. The term information culture (IC) has been used in current literature and in institutional guidelines [2]. There have been different concepts and approaches to IC [7]; some nuances pointed out by researchers are: that it is related to groups or institutions, it consists of shared values toward information-related activities [8] that "may significantly motivate information sharing incentives" [9], among others. One of the most recent references on the subject consists of a three-level model related to people's values and attitudes toward information, where the 2nd level is devoted to IL and DL and has been currently explored at a conceptual level [10]. The present understanding of IC is grounded in the cited understandings, and furthermore, concentrates on information practices and development of competences for the integration of $\mathrm{IL}, \mathrm{DL}$, as well as academic writing, communication and research competences. The following sections summarize insights into the institutional context, staff involved, objectives, structure, methods and preliminary results of the new and holistic information literacy program being developed within the System of Libraries (SL) of the three-campus CETYS Universidad in Baja California (Mexico).

\subsection{Institutional Context}

The Program 'Information Culture Development' (ICD) has been grounded in the previous working and research experiences of the 'Information and Learning Development Librarians' (ILDL) [11], who were tasked with its development, as well as in a research study based on the available literature and interviews with academic staff in order to find out about their needs. ILDL is also framed within the ideas of key institutional documents, such as the 2020 Development Plan (P2020) [2] and the Higher Education Teacher's Guide (HETG) [12]. ICD is one of the flagship projects of the SL, and the main project of the ILDL, undertaken under the HETG principle of fostering culture, reading, supporting teaching-learning processes, and being a dynamic source of knowledge [12]. ICD is directed mainly to CETYS teachers and students, but its execution will also raise new processes for staff training and reengineering of library activities related to ICD. Moreover, it will facilitate the necessary knowledge for their users to develop strategies for acquiring, appropriating and assessing information and ICTs, specifically for learning purposes. ICD is consonant with different facets of the P2020 [2], its concepts, pillars, and objectives. The concepts highlighted are: flexibility, innovation and pertinence, applied research, sustainability, accreditation, competences, community impact, results orientation, transparency, and evidence. P2020 pillars are: a) high educational quality, related to national and international accreditation; b) sustainability; c) learning community, involving learning-centered curriculum design, its measuring and the use of information in decision-making, enhancing a research culture, and information seeking and analysis, which are aspects clearly related with IL programs; d) worldwide competitiveness. Regarding P2020 objectives the SL, through their ILDL, must contribute to enhance teaching, research and extension tasks by developing teachers with the required 
competences, with a focus on learning measurement and the use of technology to support learning. Moreover, IC must be transformed and consolidated, to reflect the objective of diversifying educational offerings, emphasizing blended and online modalities, and improving information systems and resources in order to be able to deploy these learning modalities. P2020 also proposes the so called Distinctive Elements of CETYS Education (EDECS), which are: a) information culture; b) entrepreneur and innovation culture; c) internationalization; d) sustainability; and e) linkage and social responsibility. Clearly, ICD was framed within the first of these EDECS, information culture. In consequence, the program must concentrate on this element's actions that are described in the P2020, to further develop and expand upon them. These actions are related to: service capacity and variety, hiring professional staff and professionalizing nonprofessional staff in the SL to strengthen IC, to evaluate the impact of the SL initiatives. In 2013, the institution hired two highly qualified librarians, one national and one international. They were initially appointed as reference librarians, but later renamed as Information and Learning Development Librarians (ILDL). The hiring of the ILDL was proposed in P2020 as one of the actions to strengthen IC and it was among the recommendations that the Western Association of Schools and Colleges (WASC) made after granting CETYS their international accreditation. The reasoning behind the renaming was that these librarians are expected to make large contributions and have more comprehensive roles; they still provide reference services but they are teachers and lifelong learners, researchers, experts on information, technology objects and environments; they are information customizers, embedded librarians, and dynamic agents of change in the institution. They have the institutional mission of developing IC through the creation, planning, development, execution, and evaluation of the ICD. The name ILDL was partly inspired by the "Selected Academic Librarian Position Titles for Positions that Further the Teaching Mission of the Library" [13]. Although the final name is long, it was intended to be very simplistic: Librarians tasked with the Development of Information culture for Learning experiences and purposes.

\section{The Program Information Culture Development (ICD)}

ICD was built after some diagnostic interviews were conducted among academics. These interviews were not structured but only driven by some guiding questions to profile the staff interviewed, their needs and expectations regarding library services, and to identify possible allied academic staff [11], determining their academic, professional and research background, English proficiency, among other aspects. Then, their needs and expectations helped shape the first incarnations of the IL program, as they are the first intended stakeholders; thus, the ICD intends to support academic staff regarding learning through IL and DL, together with what the SL can do for them and what they want it to do for them. Furthermore, the interviews continued as conversations as the ILDL librarians talked about the input from other academics, their ideas for the IL Program and about what types of services they could provide. Staff comments for this latter part were interesting to measure the reactions toward some ideas and possibilities. Given the importance placed on participation, IL, DL, 
reflection and improvement upon learning and teaching practices, ICD is driven by the methodological tradition of action research (AR). Framing IL initiatives within AR is nothing extravagant [14, 15]; it is just something that has not been widely or properly stated by researchers and practitioners [1]. In fact, ICD's vision is that all its activities must be grounded in research $[1,10,15,16]$, because among other things, ICD seeks to position the SL as a research unit of the institution, which would feed learning processes and apply the scientific method to the SL's activities, use, and professionalize their non-professional staff. This new position of the SL, following the guidelines of P2020 [2], seeks to generate institutional conditions for academic staff to enhance research. The SL's role is to be an example of an active research community and a driving force for encouraging and supporting CETYS researchers.

The use of an AR perspective was already decided upon and kept in mind at the moment of conducting the previously mentioned diagnostic interviews. This was important for keeping a bottom-up participatory approach in the IL initiative. By having the ILDL systematize, adopt, and build upon the academic staff input, the IL initiative became a product of the learning community. Moreover, this is a primordial approach for defining the aspects related to this project and it would set a precedent and an example of a SL that participates in, enhances and promotes IC within and for an academic institution, by also seeking the active participation of academic staff. After conducting the interviews, reviewing pertinent literature, and setting the basic ideas, ICD's main elements started to be developed. ICD's aims at addressing information and digital literacy tasks, as well as provoking and supporting reflection and improvement upon other university practices related to curriculum, teaching, and research. Accordingly, ICD's objectives were stated in order to address all university stakeholders, as well as these methodological and conceptual stances. The general objective of the Program is to serve as a supporting axis to research, teaching, and learning in CETYS Universidad. Specific objectives are as follows: a) support and nurture teaching and learning practices of CETYS community through information culture and reflection; b) promote products, services, and resources of the SL and justify their increase and development through the enhancing and massively increasing their use; c) develop an information culture in the CETYS community and at the individual level develop independent and critical information users, who are able to tap into appropriate information and technological tools; d) Professionalize and enhance the staff, procedures and resources of the SL.

ICD must be an initiative from the SL, executed by the ILDL, under a close collaboration with academic staff, thus generating results and best practices with a bottom-up approach and avoiding the imposition of practices without first studying all elements at stake. Additionally, the courses of this initiative would contribute to the achievement of the above-mentioned practices, and their socialization would provide feedback to the enrichment and revision of the courses as well.

\subsection{ICD Axes and Initiatives}

ICD's initiatives, products and resources were divided into four axes, three main ones and a transversal axis. All but the latter include the development of courses and video 
tutorials derived from courses and intended for students and members of the university. The following paragraphs summarize the axes and their initiatives.

Axis I. Curriculum and Learning Support through IL and DL. It seeks to support teachers to enhance and innovate classroom practices, both those of student learning and of teaching through IL and DL. Initiatives of this axis include:

1. development of subject guides both by teachers' request and through the initiative of the ILDL, in order to strategically cover university curricula. These guides compile references of recent documents that are related to a given course available in the library, subscribed databases and bookstores, in order to provide the possibility of updating the library collection, the bibliography of the courses, and the teachers themselves

2. library-academia joint activities: product of a curricular analysis and determining a learning activity with a given teacher that integrates IC into the curriculum of their courses

3. documents offered by area of knowledge: this is a variation of the subject guide, but intended for students and made in general for every study program and not each course, they list resources available in the subscribed databases and some keywords students can use to retrieve them

4. courses of this axis: they are exclusively for academic staff and alternatively can be offered to students of the Master in education, they cover: a) recording and editing of digital audio and video, b) social media for education purposes, c) content curation and d) really simple syndication (RSS) for learning.

Axis II. Information and Digital Literacies Development. It contains the majority of courses for teachers and students about access, use and evaluation of information, and the appropriation of ICTs for learning purposes. It includes the initiatives: a) developing flyers on the SL's resources and containing a summary of course contents; b) production of promotional videos on the SL's products and services; c) courses on: how to use the SL, SL online catalog, and academic databases (AD).

Axis III. Research and Scientific Communication Support. It concentrates initiatives related to research and scientific communication and is targeted at each academic program of the institution. The following actions are included: a) promotion materials for citation styles for each field: demonstrating the different citation styles used by each field, i.e. IEEE, MLA, ISO, Chicago; b) Scientific Communication Support Guides: provided to facilitate research and lowering the barriers of entry for the publication on a given field, compiling information about publications (language, publisher, indexing, availability in the SL, manuscript types and extension, citation style, and open access policy), together with information on pertinent professional congresses, groups of interest, and professional associations; c) Courses: on citation styles; the peer review process; reference managers; Self-archiving in open Access repositories, writing an article and publishing it; AR workshops; creation and 
management of online researchers profiles in order to improve the promotion, visibility, and recognition of CETYS researchers. This latter course would contain the construction of a communication, promotion and visibility strategy by using sites such as LinkedIn, ResearchGate, Google Scholar, ORCID, among others.

Transversal Axis. Evaluation and Communication of Results. This is intended as an administrative axis, which would aim at conducting research to enrich the other axes, and evaluate and communicate the results of the initiative. It is comprised of: a) constructing data collection instruments to measure both learning and user satisfaction; b) developing procedures and formats for new services and instructional design formats; c) measurement and statistics; d) supporting IC testing through international instruments such as SAILS and iSkills; e) building a mirror SL website as a highly updated blog; f) communicating ICD's results in scientific conferences and publications.

\subsection{Development of Courses}

ICD entails the offering of courses, which are being developed with a common methodology among the ILDL. Prior to their arrival at the institution, there were already user training initiatives, however, the current effort seeks to unify and formalize a solid structure of supporting materials and learning experiences comprised of learning objectives, contents, activities, and complementary readings. This working methodology is consonant with the HETG [12], which considers an integrative student profile, so it compels teachers to use more than one pedagogical resource. The level of difficulty of the courses consists in dividing the courses into a modular structure according to learning objectives and contents and arranging them by difficulty. In this manner, the course offerings consist of the levels beginner, intermediate, and advanced. Because ICD was designed, executed and evaluated at the same time, the courses are developed in two modalities: the first we call 'remedial', proactively offered to the community but mostly facilitated by request, but that does not follow a fixed structure, because it still could not be properly developed. The second modality is 'formal', because these are courses that were properly planned and instructionally designed. The production of the courses has to be exhaustive in order to generate simultaneously, and for every topic, a regular course, an online course, a video tutorial, and the corresponding flyers or manuals of instructions. The development of courses was planned this way in order to cover all demands from different learning styles and being able to offer something to all stakeholders.

\subsection{Holistic Cycle}

ICD introduces the idea of a holistic cycle as the ideal way of presenting IC courses that are naturally related among each other. The holistic cycle comprises a reasoned and sequential articulation of courses from different axes. Despite the fact that this is more time consuming for the participant than isolated courses, the main idea is to be able to offer different combinations of stages and competences tailored for each group of stakeholders. The holistic cycle implies sessions of training, working, and reflection, 
which as AR dictates: one stage can lead to the other, as well as to the previous one, or to repeat the entire cycle once it is finished. The ideal holistic information culture cycle would contain the following stages and competences: a) Search in $A D$; b) Store and annotate through reference managers and note-taking and archiving software; c) Research and reflect by conducting an AR project in order to revise teaching or classroom practices; d) Communicate through presentations, conferences, publications, and media-editing software; and e) Promote visibility using professionally-oriented social media sites and traditional academic online indexes.

\section{Piloting the Program}

This section summarizes the methods and results from ICD's pilot stage, which consisted of two pilot studies conducted in two CETYS campuses, and involved AR-grounded training activities. Results are provided for Pilot 1 with High School Teachers (HST) in Mexicali and for Pilot 2 with undergraduate students (UGS) in Tijuana. Each pilot experimented with different forms of a holistic cycle.

Pilot 1. Mexicali. Holistic Cycle for HST. It was based on a three-stage holistic cycle for the competences: a) Searching with AD; b) Store and annotate with a reference manager (RM); and c) Research, Reflect by having an AR seminar and conducting an AR project on the HST's own classroom practices. This was planned with the High School Direction of CETYS Universidad Mexicali (CETYS includes the last years of high school in its academic offer). The cycle lasted for 20 hours, divided among an AD, a RM, an AR seminar, and the writing of the research project.

Pilot 2. Tijuana. Holistic Cycle for UGS. It was based on a two-stage holistic cycle for the competences of searching, evaluating, and citing documents from AD. 2-hour training sessions were provided to 10 groups of UGS enrolled in different programs and the examples and practical activities were tailored to each group's interests.

\subsection{Data Collection and Analysis}

Data collection instruments used were diagnostic and follow-up questionnaires. In the case of the questionnaires, each stage of the holistic cycle had diagnostic and follow-up questionnaires. These questionnaires had similar questions, which were adapted to each competence or platform being taught, in order to make the results comparable. They were intended to provide quantitative and qualitative insights to both learning and satisfaction of the participants enrolled in a holistic cycle. Questions on a diagnostic questionnaire were: a) how are you used to search / investigate / cite? b) Which uses you think you can give to APA Style /AD/AR? Both questions serve to assess learning. c) Have you actually used APA Style/AD /AR? d) Please describe your experience with APA Style/AD/AR. Both questions concentrate on satisfaction. Moreover, the follow-up questionnaire questions were: a) what have you learned in this session? b) Which uses you think you can give to this learning? Both questions 
serve to appreciate learning that took place. c) A question that used a Likert scale for rating the topics, teaching strategies, teacher, resources, and other aspects of the session; d) Please provide a positive and a negative aspect of this experience; e) Did this experience fulfilled your expectations? Please explain why or why not. These last three questions seek to assess satisfaction. Similar questions were located in the diagnostic and follow-up questionnaires in order to provide and facilitate contrasting of responses when analyzing them. Furthermore, a final questionnaire was prepared exclusively for Pilot 1 , in order to sum up the experience and provide more insights regarding participants' opinions on what they could learn during the workshops and how satisfied they were with them. In essence, the final questionnaire had questions that also intended to look at satisfaction and learning but assessing the holistic cycle as a whole, thus justifying that it was only passed among HST. Pilot 1 participants answered 3 diagnostic, 3 follow-up and the final questionnaire, while UGS in pilot 2 completed 2 diagnostic and 2 follow-up questionnaires. Furthermore, HST's AR projects were other data collection instruments used to determine the success of previous activities and to identify their strengths and weaknesses in IC-related competencies, specifically research and scientific communication competencies. Data Analysis was based on content analysis and category coding of participants' answers. Research projects were analyzed taking into account formal aspects such as: use of concepts, structure, clarity of language, use of sources and citations, methods, and quality of instruments proposed.

\subsection{Preliminary Results}

Pilot 1. Mexicali. 58 HST enrolled in this pilot study and $81 \%$ percent of them were able to present a draft of their AR project. The questionnaires allowed for profiling their information practices. Regarding the use of the tools in question: a) $44 \%$ of them used $A D$ at least once, which is a low number, considering that it is one of the resources the institution subscribes. Moreover, when asked about how they search for academic information $29 \%$ cited the Internet and $10 \%$ cited AD subscribed by the institution; b) $2 \%$ have used a RM, which is not so surprising, as this tool is still considered niche and of course most teachers still use computer folders, Internet browsers, or email to store files; and c) $25 \%$ have used the AR method, which is a good sign if we were to push an AR agenda in the institution. Regarding the research projects, teachers divided themselves in 11 groups and the ILDL received 19 versions of the projects in total to revise, which had a total of 303 pages, 182 references, and the feedback provided by the ILDL was contained in 11 general comments and 538 comments over the text of these projects. 9 of the 11 projects fit the AR tradition, 6 groups specified this in their texts, but overall all the projects are doable and worthwhile. The topics of the projects were: academic performance, learning environments, following directions, attitudes and perceptions toward learning, emotional health, critical thinking, study habits, and social media. This experience helped confirm some assumptions about the needs and practices of academic staff [6], and furthermore we identified some areas to improve their IC competences: clarity and structure for scientific communication, use of sources, citation style and organizing sources in the text 
produced; increase their use of information sources, both subscribed and those produced in the institution; clarity and precision of the language used and consonance with the methodological approach chosen. Regarding their evaluation of this experience, 26\% HST communicated that this ICD pilot is better than previously offered library courses. $17 \%$ claimed it was similar. $17 \%$ percent said it was worse, and $40 \%$ declared that this is the first library course they had attended, which still gives ICD a large user base to satisfy, because this is just a sample of CETYS teachers. 65\% HST stated they would like to attend more library courses. 3\% expressed a negative answer on the matter, while 32\% stated that 'it depends', then cited various reasons. Furthermore, they pointed out that some areas to improve ICD's courses are: having more practical activities, though some contents are necessarily theoretical; better balance between what is optional and mandatory, to have complementary readings and compulsory advisory sessions, revise durations and schedules; fragment users according to their competences and have smaller groups; encourage more collaborative work and teachers' active participation; improve computer equipment; and demonstrate more clearly the usefulness of some tools. Regarding the learning they experienced, we can say this experience was successful because HST answered that they learned about AR (33\%), AD (27\%), RM (25\%), about some improvements for their teaching practices (11\%), and about methodology in general (4\%). In addition, HST main statements on the possible uses for the learning achieved throughout the cycle, consisted in ameliorating their teaching practice (26\%), conducting research (17\%), managing a personal digital library (11\%), among others.

Pilot 2. Tijuana. 10 groups comprised by 127 UGS were trained on the use of AD and APA style. From the questionnaires we gathered that $69 \%$ have used $A D$ before. When asked about how they search for academic information $46 \%$ cited the Internet, $16 \%$ AD subscribed by the institution, and $11 \%$ books. Moreover, they listed the uses for $A D$ as to: find reliable information (43\%), locate specialized information (32\%), for research (16\%), and recreation (8\%). Their experiences using AD have been good in general (19\%); they cited challenges such as complex search and navigation (17\%), not finding what they need (13\%) and that few platforms are in Spanish (10\%). UGS stated that they learned about accessing AD (38\%), performing basic searches (20\%), creating personalized accounts (13\%), use filters to refine searches (11\%), correctly use the citation style (10\%), and retrieving trustworthy information (8\%). UGS expressed that the uses they foresee for the learning they had are: for [writing] academic papers (50\%), retrieving reputable information (17\%), downloading articles (12\%), and recreation (10\%). USG shared the desirable belief that through AD they may perform better in academic work.

\section{$4 \quad$ Concluding Remarks}

ICD has the potential of facilitating, developing, and strengthening IC in the institution, providing support for the most basic competences to the most complex ones. The ILDL are developing courses, flyers and tutorials, but also initiatives that were not 
detailed in this article, including information products such as the Subject Guides and the Scientific Communication Support Guides (SCSG). The SCSG should help lower the barriers of entry to some challenging academic activities such as scientific publishing, which has been pointed out as having an IC based on 'strong input filters' [15]. Meanwhile, and regarding basic competences there is still work to do for the ICD in order to overcome common challenges among UGS and HST such as the language barrier, making sense of the purposes of developing an institutional IC, aligning ICD with the formal Institutional Learning Results, and overcoming the limited use of the subscribed $A D$ and sources produced in the institution, thus reducing and making a more conscious use of the Internet as a source of information. Results indicate that through training sessions, the community may realize that ICD is a means to enhance teaching and learning. Moreover, its grounding on AR, the holistic cycle, axes structure, and complementary initiatives such as flyers, tutorials and innovative information products will result in powerful guidelines and means to support the development of IC in CETYS Universidad.

\section{References}

1. Machin-Mastromatteo, J., Lau, J., Virkus, S.: Participatory Action Research and Information Literacy: Revising an Old New Hope for Research and Practice. In: Kurbanoglu, S. et al (eds.), Worldwide Commonalities and Challenges in Information Literacy Research and Practice, pp. 48--53. Springer, Heidelberg (2013)

2. CETYS Universidad.: Plan de Desarrollo 2020. IENAC, Mexicali (2011)

3. Lau, J., Cortés, J.: Normas de Alfabetización Informativa para el Aprendizaje. Universidad Autónoma de Ciudad Juárez, Mexico (2004)

4. Lau, J. Guidelines on Information Literacy for Lifelong Learning. IFLA, Veracruz (2006)

5. ACRL: Research Agenda for Library Instruction and Information Literacy, http://www.ala.org/acrl/aboutacrl/directoryofleadership/sections/is/iswebsite/projpubs/researchagendalibrary

6. Lau, J., Gárate, A., Osuna, C.: Walking from Concepts to Facts: A Holistic Information Literacy Approach Experience at the University Level. In: Kurbanoglu, S., et al (eds.), Worldwide Commonalities and Challenges in Information Literacy Research and Practice, pp. 302--308. Springer, Heidelberg (2013)

7. Oliver, G.: Investigating Information Culture: A Comparative Case Study Research Design and Methods. Archival Science 4(3-4), 287--314 (2004)

8. Davenport, T.: Information Ecology. Oxford University Press, Oxford (1997)

9. Shih, H., Lai, K., Cheng, T.: Examining Structural, Perceptual, and Attitudinal Influences on the Quality of Information Sharing in Collaborative Technology Use. Information Systems Frontiers (2013)

10. Oliver, G., Foscarini, F.: Records Management and Information Culture: Tackling the People Problem. Facet Publishing, London (2014)

11. Machin-Mastromatteo, J.: Programa de Desarrollo de Competencias en el Uso de la Información de la Biblioteca Pedro Grases: Presente y Futuro. In: Lloret, N. (ed.) Nuevas Perspectivas para la Difusión y Organización del Conocimiento, Proceedings of IX Congress ISKO Spanish Chapter, pp. 169--179. Universidad Politécnica de Valencia, Valencia (2009) 
12. CETYS Universidad. Guía del Profesor de Educación Superior. IENAC, Mexicali (2012)

13. Shank, J., Dewald, N.: Academic Library Administrators' Perceptions of Four Instructional Skills. College \& Research Libraries 73(1), 78--93 (2012)

14. Vezzosi, M.: Information Literacy and Action Research: An Overview and Some Reflections. New Library World 107 (7-8), 286--301 (2006)

15. Hill, C.: Improving information skills programs using action-research. In: Booker, D. (ed.) Concept, Challenge, Conundrum: From Library Skills to Information Literacy, Proceedings of the Fourth National Information Literacy Conference, pp. 139--46. Charles Sturt University Press, Adelaide (2000)

16. Pickering, N., Crow, S., Franklin, L.: Information Literacy and Information Literacy Skills Instruction: Applying Research to Practice in the 21st Century School Library (3rd ed.). Libraries Unlimited, Santa Barbara (2011) 